\title{
Comportamiento del Hierro, Cromo, Cadmio y Plomo Total en las Aguas Superficiales del Río Manzanares, Venezuela, Durante los Periodos de Sequía y Lluvia en el Año $1994^{1}$
}

\author{
IVAn LeÓn LunA, William Senior, ${ }^{2}$ y Gregorio MartíneZ
}

\author{
Departamento de Oceanografía, Instituto Oceanográfico de Venezuela, \\ Universidad de Oriente. Av. Universidad, Cerro Colorado, A.P. 245, 6001, Cumaná, Venezuela
}

\begin{abstract}
Results are presented for total iron, chromium, cadmium, and lead concentrations measured in the surface water of the Manzanares river (Venezuela), during two typical weather periods of the region (May and November) of 1994. The existence of removal processes in the early stages of the mixing process of freshwater and seawater is evident from their spatial distributions. It is suggested that this removal of heavy metals is associated with the geochemical process of flocculation and the formation of oxihydroxides, rather than with the dilution process. Input of heavy metals to the ecosystem was observed at low salinities that are likely associated to the process of desorption and are due to anthropogenic activities.
\end{abstract}

RESUMEN.-Se presentan los resultados del análisis de las concentraciones de hierro, cromo, cadmio y plomo total en las aguas superficiales del río Manzanares, Venezuela, durante dos períodos climáticos típicos de la región (mayo y noviembre) durante 1994. La distribución de estos elementos a lo largo del gradiente salino pone en evidencia la existencia de procesos de remoción de los elementos en los primeros momentos de la mezcla entre las aguas dulces y las aguas marinas, sugiriendo que dicha mezcla es debida a procesos diferentes a los de dilución, tales como los procesos geoquímicos de floculación y la formación de oxi-hidróxidos. Igualmente se evidencian aportes a salinidades intermedias debido a procesos de desorción y actividades antropogénicas.

\section{INTRODUCCION}

El río Manzanares está ubicado en una zona tropical caracterizada por la existencia de un período de sequía, que va de diciembre a junio, y un período lluvioso, desde julio a noviembre. Su vertiente está situada a 1,400 metros sobre el nivel del mar en la serranía del Turimiquire en la costa nor-oriental de Venezuela, desembocando en el Mar Caribe a la entrada del Golfo de Cariaco (Fig. 1). Su hoya hidrográfica cubre una extensión aproximada de 1,652 kilómetros y la escorrentía global podría estimarse en 750 millones de metros cúbicos. El río recibe aguas que drenan aproximadamente 5 mil hectáreas de cultivos de caña de azúcar y los desechos urbanos e industriales de más de 400 mil habitantes distribuidos entre las ciudades de Cumanacoa y Cumaná. Más detalles sobre el área de es-

\footnotetext{
${ }^{1}$ Este trabajo forma parte de la Tésis de Maestría en Ciencias Marinas del Lic. Ivan León Luna.

${ }^{2}$ A quien debe ser dirigida toda correspondencia (INTERNET:wsenior@sucre.udo.edu.ve).
}

tudio son descritos en Maloney (1966), para su geología; Lemus y Bastardo (1972), Fernández (1971, 1973, 1984), para la contaminación bacteriológica; Alvarado (1976, 1979), Godoy (1991) para la contaminación orgánica, Senior y Godoy (1990) para la distribución de los elementos nutritivos en la cuenca baja del río Manzanares, y Senior (1994) para la evaluación ambiental del ecosistema.

En vista del interés reinante por la contaminación por metales pesados de los sistemas estuarinos y zonas litorales, se presentan los resultados obtenidos en las aguas superficiales del río Manzanares, Venezuela, durante los meses de mayo y noviembre, los cuales mostraron las situaciones extremas durante el año estudiado.

\section{MATERIAles y MetOdos}

Veintidós muestras de agua superficial fueron colectadas en cada uno de los cruceros realizados al río, presentándose particularmente los resultados obtenidos durante los meses de mayo (época seca) y no- 


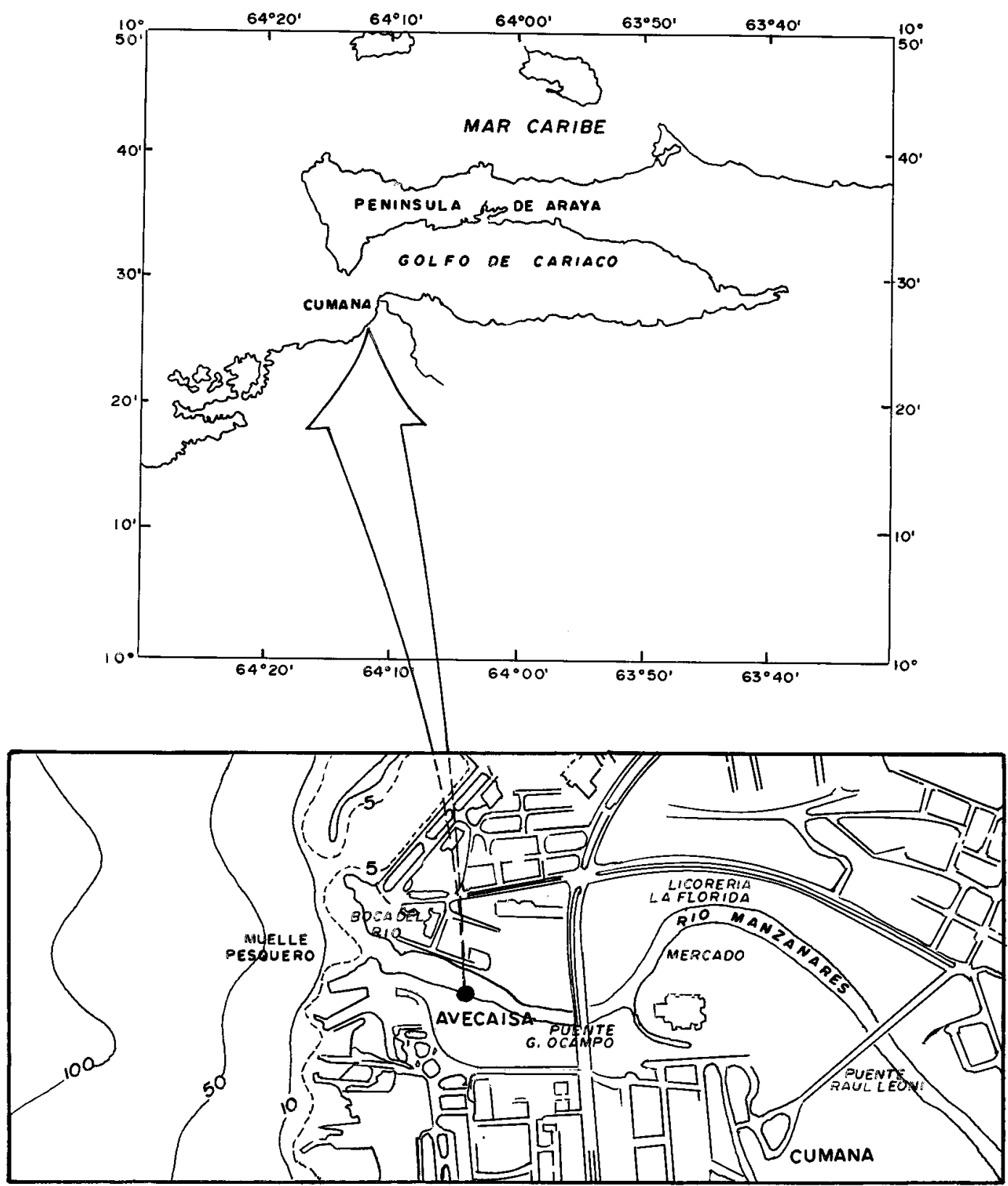

FIG. 1. Area de estudio. Las muestras de agua superficial fueron colectadas según las variaciones de salinidad con la finalidad de cubrir todo el gradiente salino.

viembre (época de lluvia) de 1994. La toma de muestra se realizó siguiendo el gradiente salino, desde 0 psu (puente G. Ocampo) hasta salinidades superiores a 36 psu (cercanía de la isobata de $100 \mathrm{~m}$ ), en dirección a la Península de Araya (Fig. 1).

Todo el material de vidrio y plástico des- tinado para la toma de muestras fue previamente lavado con ácido nítrico al 10\% $\mathrm{v} / \mathrm{v}$. Las muestras fueron colectadas mediante la utilización de un envase plástico de 10 litros de capacidad. Un litro de cada muestra fue transferido a botellas de poliestireno y acidificado con $5 \mathrm{ml}$ de ácido 
TABLA 1. Porcentaje de recuperación y precisión de los análisis de metales.

\begin{tabular}{ccc}
\hline \hline Metal & $\begin{array}{c}\text { \% de } \\
\text { recuperación }\end{array}$ & $\begin{array}{c}\text { Precisión } \\
(\%)\end{array}$ \\
\hline $\mathrm{Cd}$ & 94.9 & 2.03 \\
$\mathrm{Cr}$ & 93.0 & 4.30 \\
$\mathrm{Fe}$ & 97.1 & 1.41 \\
$\mathrm{~Pb}$ & 93.0 & 1.85 \\
\hline
\end{tabular}

nítrico concentrado y congelado $\left(-20^{\circ} \mathrm{C}\right)$ hasta el momento del análisis.

La salinidad fue estimada in situ (YSI modelo 33), y luego verificada en el laboratorio con un salinómetro de inducción Kahlsico modelo 118 WC 200 con una precisión de $\pm 0.001 \mathrm{psu}$. La temperatura y el $\mathrm{pH}$ fueron medidos in situ con un termómetro de mercurio con precisión de $\pm 0.1^{\circ} \mathrm{C}$ y un pH-metro Bantex LCGS5 con una precisión de \pm 0.01 unidades, equipado de una sonda ATC. El material en suspensión se retuvo sobre filtro de fibra de vidrio Gelman Science tipo A/E $47 \mathrm{~mm}$.

Los metales pesados fueron determinados en agua no filtrada y quelatados con Pirrolidina Dithiocarbamato de Amonio (APDC) y extraídas con Metil Isobutil Cetona (MIBK).

Los quelatos de metales en fase orgánica fueron digestados con ácidos nítrico y sulfúrico concentrados para obtenerlos en fase acuosa, según las recomendaciones de Olsen y Sommerfeld (1973) y el Standard Methods (1992). Los patrones y los blancos fueron sometidos a las mismas condiciones de las muestras. Estos últimos se prepararon utilizando agua estuarina previamente extraída.

El contenido de metales totales fue cuantificado en un espectrofotómetro de absorción atómica Perkin-Elmer modelo 3110 con llama aire-acetileno y corrector de fondo de deuterio. Las longitudes de onda utilizadas fueron 248.3, 357.9, 228.8, y $283.3 \mathrm{~nm}$ para $\mathrm{Fe}, \mathrm{Cr}, \mathrm{Cd}$, y $\mathrm{Pb}$ respectivamente. En la Tabla 1 se presentan los resultados del porcentaje de recuperación de cada uno de los metales examinados, así como la precisión obtenida mediante la determinación de cuatro réplicas de dos muestras diferentes.

\section{Resultados}

Los resultados de temperatura, salinidad, pH y material en suspensión (MES) son mostrados en la Tabla 2. Los valores de temperatura en mayo estuvieron comprendidos entre 29,2 y $24,5^{\circ} \mathrm{C}$ con los mayores valores en el extremo fluvial y los menores en el extremo marino. Esta situación se invierte durante las observaciones realizadas en noviembre, cuando las temperaturas mínimas de $25,0^{\circ} \mathrm{C}$ fueron registradas en el extremo fluvial y las máximas de $29,1^{\circ} \mathrm{C}$ en el extremo marino. De manera general, los valores de $\mathrm{pH}$ se incrementan con la salinidad; se mantienen por debajo de 7 en la mayor parte del ecosistema, en mayo, alcanzando un máximo de 7.21 en el extremo marino. Durante el mes de noviembre, los valores de $\mathrm{pH}$ se aumentan alcanzando 8.01 unidades a salinidades superiores a 36.5 psu.

Las concentraciones de material en suspensión oscilaron entre 3 y $17 \mathrm{mg} / 1$ en el mes de mayo, para luego incrementarse apreciablemente durante el muestreo de noviembre cuando alcanzan valores superiores, por un factor superior a 50, a los registrados en el período de sequía. La figura 2 presenta los diagramas de mezcla metales-salinidad. La relación del hierro total con respecto a la salinidad mostró una disminución de las concentraciones para el mes de mayo entre 0 y 3 psu de salinidad, cuando pasaron de $7.5 \mu \mathrm{M}$ a $2.5 \mu \mathrm{M}$ para luego alcanzar un valor máximo cercano a $15 \mu \mathrm{M}$ en las cercanías de 10 psu. A partir de aquí, los valores disminuyeron bruscamente, entre los valores de salinidad $10 \mathrm{y}$ 15 psu y oscilaron levemente en el resto del gradiente salino, obteniéndose los registros mínimos en el extremo marino del ecosistema, donde las concentraciones fueron cercanas a los $0.2 \mu \mathrm{M}$. Así, para el hierro se registró una "pérdida" en los primeros momentos de mezcla de las aguas dulces con las aguas marinas, como consecuencia de los cambios físico-químicos que tienen lugar en esta interfase. Avanzando en la dirección positiva del gradiente salino se detectó un aporte, siendo más notable a salinidades intermedias, comportándose de una manera conservativa. Esta evidencia es 
TABLA 2. Valores de salinidad (psu), temperatura $\left(\mathrm{T}^{\circ} \mathrm{C}\right), \mathrm{pH}$ y material en suspensión (MES; mg/L), durante los dos estudios efectuados en las aguas superficiales del río Manzanares, Venezuela.

\begin{tabular}{|c|c|c|c|c|c|c|c|c|}
\hline Estación & $\begin{array}{c}\text { S Psu } \\
\text { May } 94\end{array}$ & $\begin{array}{c}\text { S psu } \\
\text { Nov } 94\end{array}$ & $\begin{array}{c}\mathrm{T}^{\circ} \mathrm{C} \\
\text { May } 94\end{array}$ & $\begin{array}{c}\mathrm{T}^{\circ} \mathrm{C} \\
\text { Nov } 94 \\
\end{array}$ & $\begin{array}{c}\mathrm{pH} \\
\text { May } 94\end{array}$ & $\begin{array}{c}\mathrm{pH} \\
\text { Nov } 94\end{array}$ & $\begin{array}{c}\text { MES } \\
(\mathrm{mg} / \mathrm{L}) \\
\text { May } 94\end{array}$ & $\begin{array}{c}\text { MES } \\
(\mathrm{mg} / \mathrm{L}) \\
\text { Nov } 94 \\
\end{array}$ \\
\hline 1 & 0.315 & 0.120 & 29.2 & 25.0 & 6.59 & 6.97 & 14.4 & 1.124 \\
\hline 2 & 0.798 & 0.123 & 29.0 & 25.0 & 6.56 & 6.94 & 8.4 & 1.102 \\
\hline 3 & 1.683 & 0.162 & 28.8 & 25.2 & 6.23 & 6.36 & 6.8 & 1.130 \\
\hline 4 & 2.500 & 0.297 & 28.8 & 25.1 & 6.50 & 6.89 & 6.8 & 958 \\
\hline 5 & 3.727 & 1.539 & 28.7 & 25.4 & 6.68 & 6.87 & 7.2 & 804 \\
\hline 6 & 5.845 & 3.298 & 28.4 & 25.6 & 6.68 & 6.90 & 10.0 & 704 \\
\hline 7 & 8.125 & 6.902 & 28.2 & 26.2 & 6.66 & 7.58 & 8.8 & 590 \\
\hline 8 & 9.956 & 8.451 & 28.0 & 25.8 & 6.68 & 7.57 & 10.4 & 656 \\
\hline 9 & 12.677 & 9.689 & 27.4 & 26.3 & 6.78 & 7.61 & 15.6 & 676 \\
\hline 10 & 13.716 & 11.584 & 27.5 & 26.6 & 6.70 & 7.63 & 12.0 & 428 \\
\hline 11 & 15.407 & 11.976 & 26.9 & 26.3 & 6.84 & 7.67 & 9.2 & 608 \\
\hline 12 & 17.373 & 15.349 & 26.5 & 28.8 & 6.84 & 7.72 & 10.8 & 164 \\
\hline 13 & 18.826 & 15.912 & 26.2 & 26.6 & 6.82 & 7.75 & 12.8 & 368 \\
\hline 14 & 21.448 & 16.997 & 26.1 & 26.8 & 6.86 & 7.80 & 13.2 & 392 \\
\hline 15 & 24.209 & 18.011 & 26.3 & 27.0 & 6.69 & 7.80 & 16.8 & 208 \\
\hline 16 & 26.531 & 20.216 & 25.6 & 27.9 & 6.93 & 7.80 & 16.0 & 148 \\
\hline 17 & 28.114 & 21.276 & 25.2 & 28.8 & 6.84 & 7.84 & 11.6 & 36 \\
\hline 18 & 30.051 & 25.304 & 24.9 & 26.9 & 6.92 & 7.89 & 14.4 & 304 \\
\hline 19 & 33.287 & 26.946 & 24.6 & 28.0 & 7.01 & 7.90 & 10.8 & 104 \\
\hline 20 & 35.144 & 28.640 & 24.5 & 27.2 & 6.97 & 7.92 & 8.8 & 210 \\
\hline 21 & 36.899 & 36.431 & 24.5 & 28.8 & 7.10 & 7.98 & 4.4 & 8 \\
\hline 22 & 36.905 & 36.599 & 24.7 & 29.1 & 7.21 & 8.01 & 3.2 & 9 \\
\hline
\end{tabular}

característica del ecosistema durante el período de sequía.

Durante la época de lluvias, las concentraciones de este elemento se incrementaron marcadamente, registrándose a nivel del agua dulce concentraciones cercanas a los $25 \mu \mathrm{M}$. Durante este período, el río constituyó la principal fuente de hierro al ecosistema, si lo comparamos con los resultados obtenidos durante el resto de los muestreos, donde se apreció siempre un aporte del elemento a salinidades intermedias, tal como lo muestran los resultados obtenidos durante el período de sequía. El diagrama de mezcla cromo-salinidad presenta características similares a las del hierro. En mayo, las concentraciones en el extremo dulce fueron cercanas a $0.06 \mu \mathrm{M}$, apreciándose una "pérdida" del elemento entre las salinidades de 0 y 2.5 psu. Las concentraciones se incrementaron luego, para disminuir progresivamente hasta no ser detectadas a partir de salinidades de 24 psu. En el período lluvioso, las concentraciones aumentaron a nivel del agua dulce hasta 0.17 $\mu \mathrm{M}$ y disminuyeron pronunciadamente hasta salinidades de 22 psu. A partir de este último punto la disminución de las concentraciones fue menos marcada, pudiéndose detectar valores de $0.005 \mu \mathrm{M}$ en el extremo marino.

El comportamiento del cadmio es muy diferente. En efecto, este elemento presenta durante la época de sequía concentraciones de $0.08 \mu \mathrm{M}$ en el extremo fluvial del ecosistema. Sus concentraciones disminuyeron marcadamente, para alcanzar valores de $0.02 \mu \mathrm{M}$ a $1.6 \mathrm{psu}$ de salinidad. A partir de aquí los valores se incrementaron para luego oscilar entre 0.04 y $0.01 \mu \mathrm{M}$ en el resto del ecosistema. Durante el período lluvioso las concentraciones en el extremo dulce disminuyen a $0.04 \mu \mathrm{M}$. Se apreció igualmente una remoción del elemento entre 0 y 1.6 psu. A partir de este punto los valores se incrementaron alcanzando concentraciones similares a las originales, cuyos valores disminuyeron posteriormente en la zona de mezcla de las aguas dulces con las aguas 

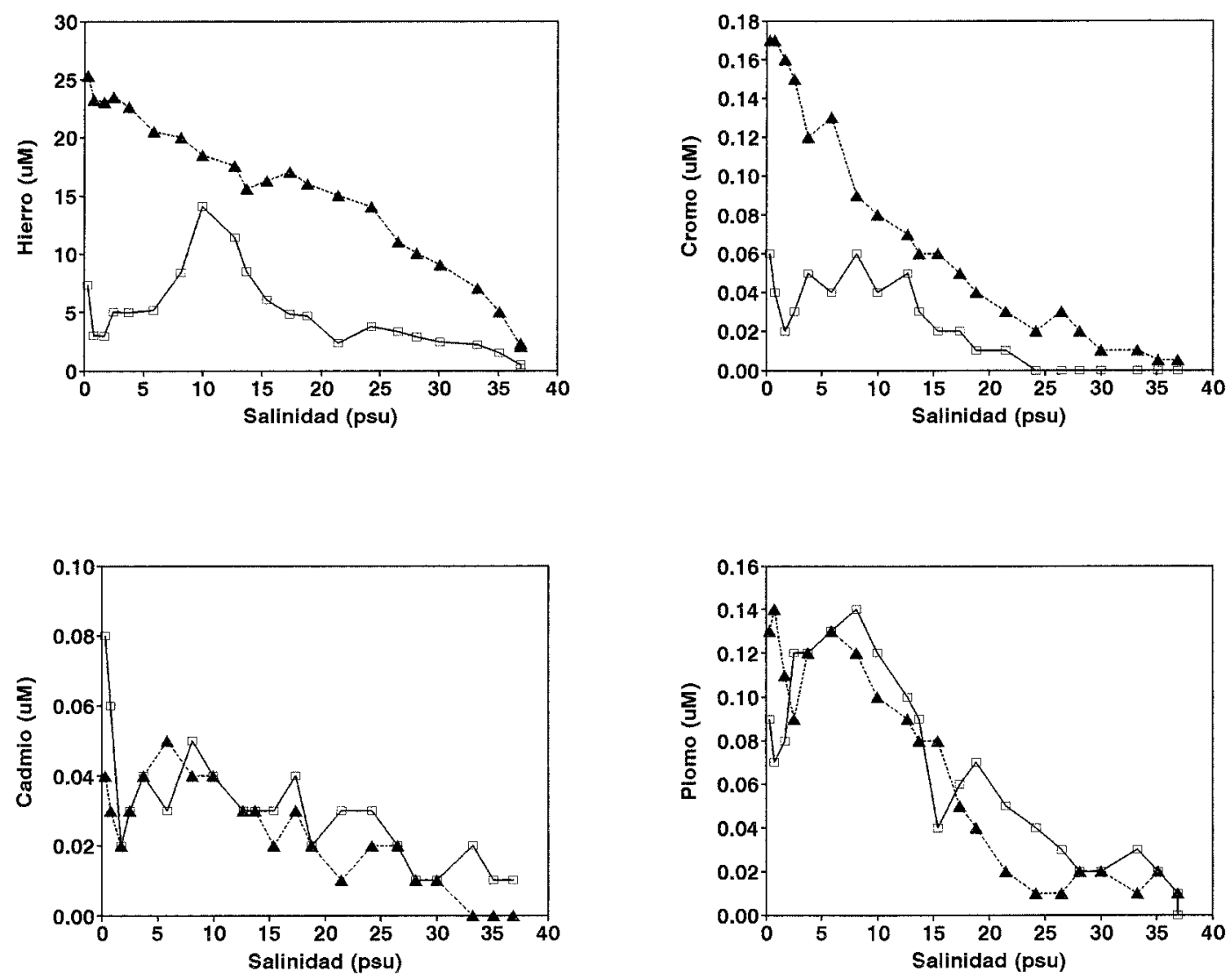

FIG. 2. Diagramas de mezcla hierro, cromo, cadmio y plomo en función de la salinidad en las aguas superficiales del río Manzanares, Venezuela, durante los meses de mayo (rectángulos) y noviembre (triángulos) de 1.994. Las concentraciones de los metales se expresan en $\mu \mathrm{M}$, mientras que la salinidad se expresa en psu.

marinas, llegando a no detectarse el elemento a salinidades superiores a 33 psu.

El diagrama de mezcla plomo-salinidad muestra para el mes de mayo una disminución de las concentraciones en los primeros momentos de la mezcla de las aguas dulces con las aguas marinas, para luego incrementarse entre las salinidades de 2.5 y $10 \mathrm{psu}$, alcanzando concentraciones de 0.14 $\mu \mathrm{M}$, valores superiores a los detectados en el extremo fluvial. Este incremento de las concentraciones de plomo sugiere que son los procesos físico-químicos que se suceden en los primeros momentos de la mezcla los que contribuyeron en mayor parte al aporte de este elemento. Posteriormente, las concentraciones presentaron tendencia conservativa. En la época de lluvia, las concentraciones de plomo en el río se incrementaron ligeramente, alcanzando valores de 0.13 $\mu \mathrm{M}$, disminuyendo igualmente durante los primeros momentos de la mezcla de las masas de agua, para luego incrementarse entre las salinidades de 3 y 6 psu. Las concentraciones disminuyeron entonces como consecuencia del proceso de mezcla hasta salinidades de $25 \mathrm{psu}$, a partir de la cual oscilaron en un estrecho intervalo (0.01$0.02 \mu \mathrm{M})$, hasta salinidades de 36 psu. Durante este período, el río constituyó la principal fuente de plomo al ecosistema, pudiéndose apreciar, sin embargo, los aportes a bajas salinidades que pudieran tener como origen la desorción del mineral desde los sedimentos.

\section{DISCUSION Y CONCLUSIONES}

Las temperaturas más bajas apreciadas en la zona marina durante el mes de mayo están asociadas a la surgencia costera que 
afecta a la región (Senior y Godoy, 1990; Godoy, 1991). El relajamiento de este afloramiento de aguas sub-superficiales durante la segunda parte del año favorece el calentamiento de las aguas marinas superficiales. Así mismo, las aguas del río sufren una baja de la temperatura como consecuencia de precipitaciones en la parte alta del ecosistema.

Los valores mínimos de $\mathrm{pH}$ en las aguas dulces y en la zona de mezcla son consecuencia, en gran parte, de los procesos de remineralización de la materia orgánica vertida en esta región del ecosistema (Godoy, 1991). El incremento de los valores de $\mathrm{pH}$ durante el período de lluvias está relacionado con la disminución del tiempo de residencia de las aguas en la zona de mezcla.

El incremento del material en suspensión durante el período lluvioso está asociado a la erosión de los suelos en la cuenca alta del río Manzanares. En este sentido Aguilera et al., (1985) señalan que la tala y quema de árboles es una de las actividades que más se realizan en esta región del ecosistema.

El hierro y el cromo muestran un comportamiento similar en la región estudiada. La correlación positiva entre ambos metales en mayo $(r=0.71)$ y noviembre $(r=0.90)$ sugiere que son afectados por los mismos procesos físico-químicos. Ambos elementos presentan una mayor remoción durante la época de sequía (60\% para el hierro y $67 \%$ para el $\mathrm{Cr}$ ), haciéndose menos notorio este proceso durante el período de lluvias. De acuerdo con Martin et al. (1993), los cambios de $\mathrm{pH}$ y salinidad estarían provocando la precipitación de oxi-hidróxidos coloidales de hierro, y adheridos a estos precipitaría los compuestos de cromo. Sadiq (1992) señala que el cromo muestra una marcada tendencia a ser adsorbido a bajas salinidades, especialmente en la superficie de los óxidos de hierro. Igualmente, estos dos elementos presentaron sus máximas concentraciones durante el período lluvioso, lo cual sugiere un aporte principalmente terrígeno. Esta situación vendría a ser confirmada por la correlación altamente significativa entre el cromo y el hierro con el material en suspensión $(r=+0.89$ para el Fe y $r=+0.95$ para el Cr) y la de ambos parámetros con el gasto del río $(r=+0.73$ y $r=+0.75$, para el hierro y cromo respectivamente). El incremento de los niveles de hierro y cromo durante el mes de noviembre podría estar enmascarando los aportes que se dan en la zona intermedia del ecosistema.

El cadmio, al igual que el resto de los metales pesados aquí estudiados, mostró un comportamiento no conservativo, remoción a bajas salinidades, oscilando ésta entre 50 y $75 \%$, y aportes a salinidades intermedias y altas. Estos resultados se asemejan notoriamente a los reportados por Sharp et al. (1982) para el estuario de Delaware y Apte et al. (1990) para el estuario de Severn. Las concentraciones de $\mathrm{Cd}$ total muestran una relación inversa con el caudal del río $(r=$ -0.72), presentando las mayores concentraciones en la época seca. Esto sugiere que la fuente principal de este elemento son las actividades antrópicas que tienen lugar en la cuenca baja del río; sin embargo, no se descarta que los procesos de desorción desde el sedimento sean otra causa de estos aportes.

El plomo total presenta un comportamiento no conservativo con aportes en la zona de mezcla entre salinidades de 0 y 10 psu que se hace conservativo hasta disminuir considerablemente a altas salinidades $(>20 \mathrm{psu})$. La región estudiada soporta un pesado tráfico de embarcaciones y en la desembocadura del río están emplazada una estación de gasolina y un muelle pesquero donde atracan barcos atuneros y de pesca de arrastre, los cuales probablemente están jugando un papel preponderante en las concentraciones apreciadas de este elemento.

Agradecimientos.-Los autores agradecen al personal Técnico del Departamento de Oceanografía del Instituto Oceanográfico de Venezuela (IOV) de la Universidad de Oriente que colaboró en la realización del presente estudio. Igualmente se agradecen las sugerencias aportadas por el Dr. Humberto González del IIT-CIMAB, Cuba y el Dr. Julian Castañeda del IOV, así como las observaciones aportadas por dos árbitros anónimos. Esta investigación contó con el 
apoyo financiero del Consejo de Investigación de la UDO, a través de los Proyectos de Investigación CI-5-019-00624/94 y CI-5019-00622/94.

\section{Literatura CitADA}

Aguilera, D. L., Lastra, R. Betancourt y M. Amundarain. 1985. Rescate del Manzanares. Informe Com. Consv. Mejor. Defen. Río Manzanares. 46 pp.

Alvarado, E. 1976. Algunas observaciones sobre las descargas de nitrógeno del río Manzanares, $\mathrm{Cu}-$ maná, Venezuela. Tesis. MsC. en Ciencias Marinas. Inst. Oceanogr. de Venezuela. UDO. 53 pp.

- 1979. Algunas observaciones sobre la concentración de fosfato en el río Manzanares. Tesis. Lic. Educ. mención química. Escuela de Humanidades y Educación. UDO 34 pp.

Apte, S., M. Gardner, A. Gunn, J. Ravenscroft y J. Vale. 1990. Trace metals in the Severn estuary: A reappraisal. Mar. Poll. Bull. 21(8):393-396.

Fernández, E. 1971. Algunos aspectos sobre la contaminación del río Manzanares por desechos industriales. IX reunión de Lab. Mar. Ins. del Caribe, Cumaná. Resumen. 19 pp.

- 1973. Algunas observaciones sobre la contaminación de las aguas costeras en la ciudad de $\mathrm{Cu}$ maná, Venezuela. Bol. Inst. Oceanogr. UDO. 12(1): 23-32.

- 1984. Contaminación de los ríos Guasdua y Manzanares, Edo Sucre, Venezuela. Bol. Inst. Oceanogr. UDO. 12(1):23-32.

Godoy, G. 1991. Estudio espacio-temporal de los par- ámetros físicos, químicos y biológicos en la zona estuarina del río Manzanares (Cumaná-Venezuela). Tesis MsC. Ciencias Marinas. Inst. Oceanogr. de Venezuela. UDO. 185 pp.

Lemus, R. y J. Bastardo. 1972. Enterobacterias de las aguas del río Manzanares de Cumaná, Edo. Sucre, Venezuela. Bol. Inst. Oceanogr. UDO. 11(2):107114.

Maloney, A. 1966. El delta del río Manzanares. Pasado, presente y futuro. Lagena. 10:3-6.

Martin, J., D. Guan, F. Elbaz-Poulichet, A. Thomas y V. Gordeev. 1993. Preliminary assesment of the distributions of some trace elements $(\mathrm{As}, \mathrm{Cd}, \mathrm{Cu}, \mathrm{Fe}$, $\mathrm{Ni}, \mathrm{Pb}$ and $\mathrm{Zn}$ ) in a pristine aquatic environment: The Lena river estuary (Russia). Mar. Chem. 43: 185-199.

Olsen, R. y M. Sommerfeld. 1973. A technique for extraction and storage of water samples for $\mathrm{Mn}, \mathrm{Cd}$ and $\mathrm{Pb}$ determination by atomic absorption spectroscopy. Atomic Absorption Newsletter. 12(6):165168.

Sadiq, M. 1992. Toxic metal chemstry in marine environments. Marcel Dekker, Inc. 389 pp.

Senior, W. 1994. Diagnóstico ambiental del río Manzanares. Inf. Téc. Dpto. De Oceanografía. IOVUDO. 22 pp.

— y G. Godoy. 1990. Estudio físico-químico del río Manzanares, Cumaná-Venezuela. Bol. Inst. Oceanogr. UDO. 29(1-2):160-172.

Sharp, J., Ch. Culberson y T. Church. 1982. The chemistry of the Delaware estuary. General considerations. Limnol. Oceanogr. 27(6):1015-1028.

Standard Methods for the Examination of Water and Wastewater. 1992. In A. Greenberg, L. Clesceri y A. Eaton (eds.), 18th ed. 